\title{
Alteration of fatty acid oxidation by increased CPT1A on replicative senescence of placenta-derived mesenchymal stem cells
}

\author{
Jin Seok ${ }^{1 \dagger}$, Hyun Sook Jung ${ }^{1 \dagger}$, Sohae Park ${ }^{1}$, Jung Ok Lee ${ }^{2}$, Chong Jai Kim³ and Gi Jin Kim ${ }^{1 *}$ (D
}

\begin{abstract}
Background: Human placenta-derived mesenchymal stem cells (PD-MSCs) are powerful sources for cell therapy in regenerative medicine. However, a limited lifespan by senescence through mechanisms that are well unknown is the greatest obstacle. In the present study, we first demonstrated the characterization of replicative senescent PDMSCs and their possible mitochondrial functional alterations.

Methods: Human PD-MSCs were cultured to senescent cells for a long period of time. The cells of before passage number 8 were early cells and after passage number 14 were late cells. Also, immortalized cells of PD-MSCs (overexpressed hTERT gene into PD-MSCs) after passage number 14 were positive control of non-senescent cells. The characterization and mitochondria analysis of PD-MSCs were explored with long-term cultivation.

Results: Long-term cultivation of PD-MSCs exhibited increases of senescent markers such as SA- $\beta$-gal and p21 including apoptotic factor, and decreases of proliferation, differentiation potential, and survival factor. Mitochondrial dysfunction was also observed in membrane potential and metabolic flexibility with enlarged mitochondrial mass. Interestingly, we founded that fatty acid oxidation (FAO) is an important metabolism in PD-MSCs, and carnitine palmitoyltransferase1A (CPT1A) overexpressed in senescent PD-MSCs. The inhibition of CPT1A induced a change of energy metabolism and reversed senescence of PD-MSCs.
\end{abstract}

Conclusions: These findings suggest that alteration of FAO by increased CPT1A plays an important role in mitochondrial dysfunction and senescence of PD-MSCs during long-term cultivation.

Keywords: Placenta-derived mesenchymal stem cell, Senescence, Mitochondria, Fatty acid, CPT1A

\section{Background}

Human mesenchymal stem cells (hMSCs) are adult multipotent stem cells that can be isolated from various tissues, including the bone marrow, adipose tissue, muscle, and placenta, which are currently considered as a powerful source for stem cell transplantation in the field of regenerative medicine [1]. Human placenta-derived mesenchymal stem cells (PD-MSCs) obtained from fetal tissue origin have emerged as a new alternative source of MSCs, which

\footnotetext{
* Correspondence: gjkim@cha.ac.kr

${ }^{\dagger}$ Jin Seok and Hyun Sook Jung contributed equally to this work.

'Department of Biomedical Science, CHA University, 689, Sampyeong-dong,

Bundang-gu, Seongnam-si, Gyeonggi-do, Republic of Korea

Full list of author information is available at the end of the article
}

have advantages for multipotent differentiation, strong immunosuppressive properties, and easily accessible to obtain abundant cells in vitro, moreover free from ethical concerns [2]. However, cultured primary human cells, including hMSCs, undergo a limited number of cell division and reach a state of irreversible growth arrest in a process called cellular senescence [3]. Senescence of MSCs is considered to be the major disadvantage of cell-based therapy. The major characteristics or molecular changes of senescent MSCs are the telomere shortening or dysfunction, DNA damage foci, enlarged cell size including flattened appearance, increased senescence-associated $\beta$-galactosidase (SA- $\beta-$ Gal) activity, oxidative stress, deranged mitochondrial metabolism, as well as altered signaling of sirtuins,

(C) The Author(s). 2019 Open Access This article is distributed under the terms of the Creative Commons Attribution 4.0 International License (http://creativecommons.org/licenses/by/4.0/), which permits unrestricted use, distribution, and 
insulin/insulin-like growth factor-1 (IGF-1) [4, 5]. In addition to these markers, increased autophagic vacuole with enhanced $\beta$-Gal activity is associated with senescent fibroblast [6]. A previous study demonstrated autophagy is activated during long-term MSC culture and autophagic activity is a requirement for maintaining the senescent state of MSCs [7]. Moreover, the biological phenomena of autophagy are similar to those of senescence including DNA damage, telomere shortening, and induction of p53 and p21 involving the generation of reactive oxygen species (ROS) in chemotherapy-induced senescence $[8,9]$.

Mitochondria are double membrane-bound dynamic organelles and generate ATP through oxidative phosphorylation as an important source of cellular energy. Mitochondrial ATP synthesis is coupled with respiration, which also produces a certain amount of reactive oxygen species (ROS), including hydrogen peroxide, superoxide, hydroxyl radicals, and singlet oxygen, as byproducts of the normal cellular metabolism [10]. Excess of ROS can cause DNA damage, lipid peroxidation, and oxidative modification of proteins, which are detrimental to cell function [11]. Thus, proper mitochondrial function is an important role in maintaining homeostasis and engagement of appropriate stress responses both at the level of the cell and the entire organism. Recently, a number of studies associated with mitochondrial metabolism or dysfunction-related stem cell biology have risen, especially in aging or self-renewal ability of stem cells [10, $12,13]$. Interestingly, enlarged cell morphology reflecting increased cell mass, one of the characteristics of senescent cells, was accompanied by increased membrane lipid content and lipid biosynthesis in both stressinduced senescence and replicative senescence [14].

Carnitine palmitoyl transferase 1 (CPT1) is a transmembrane enzyme of the mitochondrial outer membrane, which converts long-chain fatty acyl-CoA to long-chain acylcarnitine, following which carnitine acylcarnitine translocase transports the fatty acid across the inner mitochondrial membrane and then enter fatty acid $\beta$-oxidation. CPT1 is inhibited by malonyl-CoA, an intermediate of lipogenesis, synthesized by acetyl-CoA carboxylase (ACC) [15]. There are three tissue-specific isoforms of the CPT1 family, CPT1A (liver form), CPT1B (muscle form), and CPT1C (brain form) [16]. Quijano et al. demonstrated that oncogene-induced senescence (OIS) cells exhibited a marked elevation in fatty acid oxidation (FAO), and knockdown of CPT1A expression reduced oxygen consumption and basal metabolic rate of OIS cells [17]. The human placenta utilizes fatty acids as a significant metabolic fuel and derives energy from FAO [17].

Currently, researches on mitochondrial dysfunction and cellular senescence have been reported in many aspects, but there have been no studies on senescent PDMSCs and their associated mitochondrial dysfunction. In this study, we analyzed the alterations in overall cellular metabolism including autophagy and mitochondrial function during replicative senescence of PD-MSCs, and specifically focused on the effect of FAO by CPT1A inhibition in the senescence process of PD-MSCs during long-term cultivation.

\section{Methods}

\section{Mesenchymal stem cell isolation and culture}

Placenta-derived mesenchymal stem cells (PD-MSCs) were isolated from the chorionic plate of placentas as described previously [18] and cultured in alpha-minimum essential medium ( $\alpha$-MEM; Hyclone, South Logan, Utah) supplemented with $1 \%$ penicillin/streptomycin (GIBCO-BRL, Langley, Oklahoma), $25 \mathrm{ng} / \mathrm{mL}$ FGF-4 (Peprotech, Rocky Hill, NJ, USA), $1 \mu \mathrm{g} / \mathrm{mL}$ heparin (Sigma-Aldrich, St. Louis, MO, USA), and $10 \%$ fetal bovine serum (FBS; GIBCO-BRL) at $37^{\circ} \mathrm{C}$ in a humid atmosphere containing $5 \% \mathrm{CO}_{2}$. The cells before passage number 8 were "early," and after passage number 14 were "late." Immortalized cells of PDMSCs after passage number 14 were obtained with human telomerase reverse transcriptase (hTERT) overexpression as a positive control of non-senescent cells.

\section{Cell treatment with etomoxir and siRNA}

The target gene was inhibited with etomoxir (Sigma-Aldrich) and CPT1A siRNA (5'-GACGUUAGAUGAAACUGAAUU3', 5'-UUCAGUUUCAUCUAACGUCUU-3') (Invitrogen Corporation, San Diego, CA, USA). In order to know the conditions, cells were seeded in a six-well plate at a density $5 \times$ $10^{4}$ cells/well. After cultured with a plate for $24 \mathrm{~h}$, the cells were treated with $200 \mu \mathrm{M}$ etomoxir and $10 \mathrm{nM}$ CPT1A siRNA for $24 \mathrm{~h}$.

\section{Differentiation into mesodermal lineages}

For in vitro differentiation into adipoblast, PD-MSCs were plated at a density of $2.5 \times 10^{4}$ cells $/ 30 \mathrm{~mm}$ dish and cultured in adipogenic induction medium containing $1 \mu \mathrm{M}$ dexamethasone, $0.5 \mathrm{mM}$ isobutyl methylxanthine (IBMX), $0.2 \mathrm{mM}$ indomethacin, $1.7 \mu \mathrm{M}$ insulin (Sigma-Aldrich), $10 \%$ FBS (GIBCO-BRL), and 1\% penicillin/streptomycin (GIBCO-BRL) with medium changes three times a week. After 21 days, PD-MSCs were fixed with $4 \%$ paraformaldehyde (PFA) and were analyzed by Oil-Red O (Sigma-Aldrich) staining to induce osteogenic differentiation, and PD-MSCs were plated at a density of $2.5 \times 10^{4}$ cells $/ 30 \mathrm{~mm}$ dish and cultured in osteogenic induction medium containing $1 \mu \mathrm{M}$ dexamethasone, $10 \mathrm{mM}$ glycerol-2-phosphate (Sigma-Aldrich), $50 \mu \mathrm{M} \mathrm{L}$-ascorbic acid 2-phosphate (Sigma-Aldrich) 10\% FBS, and 1\% penicillin/ streptomycin with medium changes three times a week. After 21 days, calcium deposits in PD-MSCs were evaluated by von Kossa staining using 5\% silver nitrate (Sigma-Aldrich) under light 
for $1 \mathrm{~h}$. The differentiated cells for osteogenic and adiogenic were marked by arrowheads.

\section{Cell proliferation assay}

The cell proliferation was measured using Ez-Cytox (WST-1 assay) cell viability assay kit (Daeil Lab Service, Seoul, South Korea). Each PD-MSCs was seeded into 96well plate $\left(2 \times 10^{3}\right.$ cells/well $)$ and cultured for $1,2,3$, and 4 days. Then $100 \mu \mathrm{l}$ of EZ-cytox solution was added to each well and incubated at $37{ }^{\circ} \mathrm{C}$ for $2 \mathrm{~h}$. After incubation, the conditioned medium was transferred to 96-well plates and the absorbance was measured by an Epoch microplate spectrophotometer (Biotek, VT, USA) at $450 \mathrm{~nm}$.

\section{Total RNA extraction and real-time PCR analysis}

Total RNA was extracted from $80 \%$ confluent cells using TRIzol reagent (Ambion, CA, USA) following the manufacturer's protocol. One microgram of total RNA was used for cDNA synthesis and first-strand cDNA was produced using Oligo dT and Superscripts III Reverse Transcriptase (Invitrogen Corporation) according to the manufacturer's instructions. To analyze the markers related to stemness, the cDNA was quantified using SYBR green (Roche Diagnostics, Indianapolis, IN, USA) in a PCR machine (Refurbished Biometra Thermal Cyclers; LabRepCo, Horsham, Pennsylvania). Primers were targeted against Oct4 (5-CCTCAC TTCACTGCACTGTA-3, 5-CAGGTTTTCTTTCCCTAG CT-3), Nanog (5- TTCTTGACTGGGACCTTGTC-3, 5-G CTTGCCTTGCTTTGAAGCA-3), Sox2 (5-CCCAGCAG ACTTCACATGT-3, 5-CCTCCCATTTCCCTCGTTTT-3 ), hTERT (5-GAGCTGACGTGGAAGATGAG-3, 5-CTTC AAGTGCTG TCTGATTCCAATG-3), and hGAPDH (5CTCCTCTTCGGCAGCACA-3, 5-AACGCTTCACCTAA TTTGCGT-3). Target sequences were amplified by using the following conditions: $95^{\circ} \mathrm{C}$ for $10 \mathrm{~min}$, followed by 40 cycles of $95^{\circ} \mathrm{C}$ for $10 \mathrm{~s}$, at $60^{\circ} \mathrm{C}$ for $20 \mathrm{~s}$. All reactions were performed in triplicate. hGAPDH was used as an internal control gene for calculation of a qRT-PCR normalization factor. Data were analyzed by the comparative CT method. To analyze the markers related to differentiation, the cDNA was amplified using Taq DNA polymerase (Solgent, Daejeon, South Korea). Primers were targeted against osteocalcin (5-GCAGCGAGGTAGTGAAGAGA-3, 5-CGATGT GGTCAGCCAACT-3), Adipsin (5-GCTGGAGTTCAGT GGTGTGA-3, 5-ACCAACCTGACGAATGTGGT-3), and GAPDH (5-TTATTATAGGGTCTGGGATG-3,5-ACACT GAGGACCAGGTTGTC-3). Target sequences were amplified by using the following cycling conditions: $95^{\circ} \mathrm{C}$ for 15 min, followed by 40 cycles of $95^{\circ} \mathrm{C}$ for $20 \mathrm{~s}, 58^{\circ} \mathrm{C}$ and $59^{\circ} \mathrm{C}$ for $40 \mathrm{~s}, 72^{\circ} \mathrm{C}$ for $1 \mathrm{~min}$, and a final extension at $72^{\circ} \mathrm{C}$ for $5 \mathrm{~min}$. PCR products were mixed with loading dye (Cha Biomed, Seongnam, South Korea) and analyzed by electrophoresis on a 1.2\% agarose gels (Lonza, Basel, Switzerland).
The agarose gels were visualized with ChemiDoc (Bio-Rad Laboratories, Hercules, CA, USA).

\section{Protein extraction and Western blotting}

Cells were lysed in a lysis buffer $(50 \mathrm{mM}$ Tris $\mathrm{pH} 7.4,150$ $\mathrm{mM} \mathrm{NaCl}, 1 \%$ Triton X-100, and 0.1\% SDS) containing protease inhibitor cocktail (Roche, IN, USA) and phosphatase inhibitor cocktail II (A.G scientific, San Diego, USA). The protein concentration in each lysate was measured using a BCA protein assay kit (Pierce, Massachusetts, USA). Equal amounts of protein $(20-50 \mu \mathrm{g})$ were separated using 6-15\% sodium dodecyl sulfate-polyacrylamide gels electrophoresis (SDS-PAGE) and transferred onto polyvinylidene difluoride membranes (PVDF; Bio-Rad Laboratories) using a trans-blot system (Bio-Rad Laboratories). The membranes were blocked with 3\% skim milk and incubated overnight at $4{ }^{\circ} \mathrm{C}$ with the following primary antibodies specific for p21, PPAR $\alpha$ (Abcam, USA), p53, ATG5-12, Bax, Bcl2 (Santa Cruz Biotechnology, USA), p-p44/42 MAPK $\left(\mathrm{Thr}^{202} / \mathrm{Tyr}^{204}\right), \mathrm{p}$-AMPK $\left(\mathrm{Thr}^{172}\right)$, AMPK, p-ACC $\left(\mathrm{Ser}^{79}\right)$, ACC, p-Akt $\left(\mathrm{Ser}^{473}\right)$, Akt, CPT1A, PI3 Kinase p110 $\alpha$, PI3 Kinase p85 (Cell Signaling Technology, USA), LC3I, II (Novus Biologicals, USA), GAPDH (Ab Frontier, South Korea), and $\alpha$-tubulin (Oncogene). Subsequently, the membranes were washed several times with TBS-T and incubated with horseradish peroxidase (HRP)-conjugated secondary antibodies (anti-mouse and anti-rabbit IgG; BioRad Laboratories) and developed with the enhanced chemiluminescence (ECL) detection reagents (Amersham plc, cataway, NJ). The intensity readings for Western blot band were measured using an Image J program $(\mathrm{NIH}$, Bethesda, Maryland). The fold change value of the intensity is a comparative value of gene expression in each late and hTERT+ groups based on the gene expression value in the early group as 1.

\section{Cell cycle analysis}

For cell cycle analysis, PD-MSCs were fixed in 70\% icecold ethanol at $4{ }^{\circ} \mathrm{C}$ overnight. Cells were then centrifuged, washed and re-suspended in $1 \mathrm{ml}$ cold phosphatebuffered saline (PBS) containing 1\% BSA and RNase $(50 \mu \mathrm{g} / \mathrm{ml})$. Subsequently, cells were stained with propidium iodide (PI; $5 \mu \mathrm{g} / \mathrm{ml}$; Sigma-Aldrich) for $15 \mathrm{~min}$ at $37^{\circ} \mathrm{C}$ in the dark. The intensity of fluorescence was analyzed by a BD FACS Vantage SE Cell Sorter (BD Bioscience Pharmingen, San Diego, CA, USA). The percentage of cells in the G1, S, and G2/M were analyzed using Cell Quest software (BD Biosciences).

\section{Senescence associated $\beta$-galactosidase (SA- $\beta$ gal) assay}

The senescence-associated $\beta$-galactosidase activity was detected using the SA- $\beta$-gal staining kit (Cell signaling, Danvers, USA) according to the manufacturer's instructions. Briefly, cells were washed with PBS and fixed for 
10-15 min in $1 \mathrm{X}$ fixative solution at room temperature. After washing with PBS, the cells were incubated overnight at $37^{\circ} \mathrm{C}$ with $1 \mathrm{X}$ SA- $\beta$-gal staining solution $(\mathrm{pH}$ 6.0). The percentage of positive cells and cell size were analyzed with a microscope via a high and digital camera (Nikon instrument, Nikon Inc., Melville, NY, USA) and image J program (NIH). Positive signals of SA-b-gal staining were marked by an arrowhead.

\section{Bioenergetic analysis using the XF24 analyzer}

To determine the extracellular acidification rate (ECAR) and oxygen consumption rate (OCR) of senescent PDMSCs, a XF24 analyzer (Seahorse Bioscience, Billerica, MA, USA) was used according to the manufacturer's protocol. PD-MSCs $\left(5 \times 10^{3}\right.$ cells/well $)$ were seeded in the XF24 cell culture plates and incubated at $37^{\circ} \mathrm{C}$ with $5 \% \mathrm{CO} 2$ for $24 \mathrm{~h}$. The four metabolic inhibitors for analyses were oligomycin, 2-deoxyglucose (2-DG), carbonyl cyanide p-(trifluoromethoxy) phenylhyrazone (FCCP), and rotenone. ECAR, an indicator for glycolysis, was measured under basal conditions followed by the addition of $10 \mathrm{mM}$ glucose, $1.0 \mu \mathrm{M}$ oligomycin, and $50 \mathrm{mM}$ 2-DG. OCR was detected under basal conditions followed by the sequential addition of oligomycin $(1.0 \mu \mathrm{M})$, FCCP $(0.5 \mu \mathrm{M})$, and rotenone $(0.5 \mu \mathrm{M})$ as an indicator of mitochondrial respiration.

\section{MitoSox Red and MitoTracker Green staining}

PD-MSCs were stained with MitoSox Red and MitoTracker Green (Invitrogen Corporation) to quantify mitochondrial superoxide production and mitochondrial content, respectively. PD-MSCs $\left(1.3 \times 10^{4}\right.$ cells/well $)$ were seeded into 24-well culture plates and washed with Hanks' balanced salt solution (HBSS). The plates were incubated with 3uM MitoSox Red (Invitrogen Corporation) and 100 nM MitoTracker Green (Invitrogen Corporation) for 40 min at $37^{\circ} \mathrm{C}$. Cells were then washed with HBSS and incubated with $1 \mu \mathrm{g} / \mathrm{ml}$ diamidino-phenylindole hydrochloride (DAPI; Sigma Aldrich) for $1 \mathrm{~min}$ at RT. Fluorescence images were obtained using a confocal microscope (Leica TCS SP5 microscope; Leica microsystems, Wentzler, German, $\times 100$ magnifications). Positive signals of targeted fluorescence were marked by an arrowhead in our data.

\section{Reactive oxygen species (ROS) measurement}

The ROS levels in cells were measured using $2^{\prime}, 7^{\prime}$ dichlorofluorescein diacetate (DCF-DA). PD-MSCs $(5 \times$ $10^{3}$ cells/well) were seeded in the 96-well cultured for $24 \mathrm{~h}$ and treated with 50uM DCF-DA for $30 \mathrm{~min}$. After the cells were washed with HBSS twice, the fluorescence intensity at $535 \mathrm{~nm}$ was measured with excitation at 485 $\mathrm{nm}$ using an Infinite 200 Microplate Reader (Tecan m200; Tecan trading, Männedorf, Switzerland).

\section{Mitochondrial membrane potential}

Mitochondrial membrane potential was determined by 5,5',6,6' 'tetrachloro-1,1',3,3' -tetraethylbenzimidazolylcarbocyanine iodide (JC-1; Invitrogen Corporation) according to the manufacturer's instructions. PD-MSCs $\left(5 \times 10^{3}\right.$ cells/well $)$ were seeded into 96 -well culture plate for $24 \mathrm{~h}$ and incubated with $2 \mu \mathrm{g} / \mathrm{ml} \mathrm{JC}-1$. The fluorescence intensity was measured at $485 / 530 \mathrm{~nm}$ for JC-1 monomer and at $535 / 590 \mathrm{~nm}$ for J-aggregates, using in an Infinite 200 Microplate Reader (Tecan m200). Results are shown as a ratio of red $(535 / 590 \mathrm{~nm})$ to green $(485 / 530 \mathrm{~nm})$ fluorescence from JC-1.

\section{Mitochondrial mass determination}

The mitochondrial mass was evaluated using $10-N$-nonylacridine orange reagent (NAO; Invitrogen Corporation) according to the manufacturer's instructions. PD-MSCs $\left(5 \times 10^{3}\right.$ cells/well $)$ were seeded into 96-well culture plates for $24 \mathrm{~h}$ and incubated with $10 \mu \mathrm{M}$ NAO for $30 \mathrm{~min}$. After the cells were washed twice with PBS, the fluorescence intensity was measured at $485 \mathrm{~nm} / 530 \mathrm{~nm}$ using in an Infinite $^{\circ} 200$ Microplate Reader (Tecan m200).

\section{ATP production assay}

The ATP levels were measured using an ATP assay Kit (Abcam, Cambridge, MA, USA) according to the manufacturer's instructions. Briefly, PD-MSCs $\left(5 \times 10^{5}\right.$ cells/ well) were seeded into $100 \mathrm{~mm}$ dishes for $24 \mathrm{~h}$. The cells were washed with cold 1 xPBS and suspended in $100 \mu$ of ATP assay buffer in ice. After centrifugation, the supernatant was collected. ATP concentration was at $570 \mathrm{~nm}$ using an Epoch microplate spectrophotometer (Biotek).

\section{Statistical analysis}

All experiments were performed at least three times. Data are expressed as means \pm standard error of mean $( \pm$ SEM). Statistical significance between early passage versus late passage and control group with or without etomoxir and CPT1A siRNA was determinate using the Student's $t$ test and Sigma plot (Systat Software Inc.). For all analyses, $P<0.05$ was considered statistically significant.

\section{Results}

Characterization of senescent PD-MSCs during long-term cultivation

To analyze the characteristics of in vitro senescent PDMSCs during long-term cultivation, we compared differences in various biological markers related to senescence among early and late passage of PD-MSCs, and hTERT+ PD-MSCs as a positive control of non-senescent or immortalized cells by human TERT gene modification in PD-MSCs. First, stem cell markers in early and late passage of PD-MSCs were identified by qRT-PCR analysis. As shown in Fig. 1a, the expression levels of stemness 


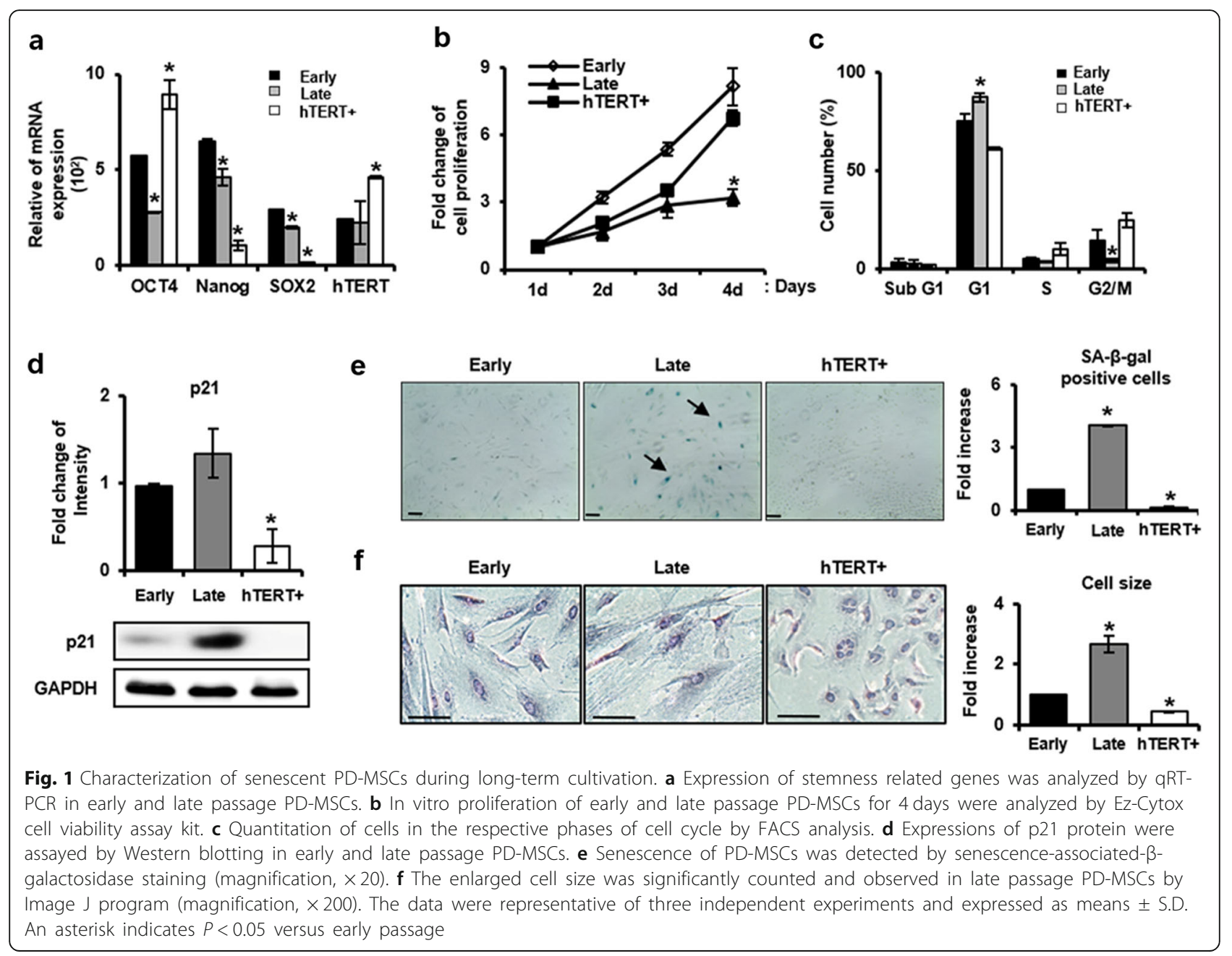

genes, OCT4, Nanog, SOX2, and hTERT showed a statistically significant decrease at late passage compared to early passage. Although Nanog and SOX2 mRNA expression was reduced, hTERT+ PD-MSCs also showed a significant increase in Oct-4 and hTERT, suggesting that immortalized PD-MSCs still retain stemness potential. In addition, a decrease in proliferation rate for 4 days was observed in PD-MSCs at late passage compared to early passage and hTERT+ cells (Fig. 1b). The cell cycle distribution of PD-MSCs was determined by FACS analysis. At late passage, the percentage of G1 cells was significantly increased up to $87.2 \%$, indicating G1 arrest, compared to early passage (75.3\%), even though no difference was observed in the percentage of sub-G1 cells in both PD-MSCs. Concomitant with this, the percentages of cells in $\mathrm{S}$ and G2/M populations were reduced from $5.1 \%$ (early) to $3.3 \%$ (late) and $14.5 \%$ (early) to $4.3 \%$ (late), respectively. However, the control hTERT+ cells showed no G1 arrest accompanied with the increase of S phase and G2/M population (Fig. 1c). We then investigated the expression level of p21, known as an inhibitor of important molecules for G1/S transition as well as typical senescence marker, at early and late passages in PD-MSCs. As expected, late PD-MSCs showed upregulation of p21 compared to early PD-MSCs, whereas p21 protein was hardly detected in hTERT+ (Fig. 1d). In contrast, the p53 expression level, known as a tumor suppressor gene and a regulator of p21, was dramatically decreased in late PD-MSCs, whereas markedly increase in hTERT PD-MSCs (Additional file 1: Figure S1). The senescent status of late PD-MSCs was confirmed by the senescence-associated $\beta$-galactosidase (SA- $\beta$-gal) staining assay showing an increased activity of the lysosomal $\beta$-galactosidase specifically in senescent cells. We also examined morphological changes of senescent PD-MSCs (Fig. 1e). In late passage PD-MSCs, the number of blue positive $\beta$-gal staining was approximately 2.67 -fold higher than in the early and cell size enlarged 4.05 -fold and flattened (Fig. 1f). However, the expression of SA- $\beta$ gal was hardly observed in hTERT+ cells and there was no change in cell morphology. To examine whether long-term in vitro culture affects the multilineage 
differentiation potential, the cells were induced to differentiate into osteocytes and adipocytes. As shown in Fig. 2a, the mRNA expressions of osteocalcin and adipsin, which are osteocyte and adipocyte markers, respectively, were enhanced significantly in early PD-MSCs after differentiation induction. However, the expression of osteocalcin was not observed in late PD-MSCs before and after induction, and adipsin mRNA expression was maintained at a constant level. Similarly, osteogenic and adipogenic differentiation potential assessed by von Kossa and Oil Red-O staining, respectively, were reduced in late PD-MSCs than early. Interestingly, the mRNA expression of osteocalcin was increased in undifferentiated condition compared to differentiated condition and the mRNA expression of adipsin was highly increased in differentiated condition compared to undifferentiated condition in hTERT + with higher self-renewal potential (Fig. 2b). Taken together, PD-MSCs during prolonged in vitro culture undergo senescence exhibiting reduced proliferation rate by G1 arrest and lowered differentiation potential, and the overexpression of hTERT helps PD-MSCs escaping from replicative senescence.
Effect of cell survival and death pathway in senescent PDMSCs during long-term cultivation

Activation of Akt and ERK1/2 plays an important role in the regulation of cell survival and proliferation. To determine the effects of survival proteins during long-term cultivation of PD-MSCs, the expression and phosphorylation levels of Akt and ERK1/2 were determined using Western blot analysis. As shown Fig. 3a, phosphorylated-AKT(pAKT)/AKT, a critical regulator of cell survival, represented a significantly decreased level in late passage compared to early and hTERT+ $(P$ value $=0.006)$. In addition, the expression of p-ERK $1 / 2$ was significantly decreased in late passage compared to early and hTERT+ PD-MSCs $(P$ value $=0.04$ ). Therefore, gene expression related to proliferation and survival was significantly declined in late passage compared to early and hTERT + .

Next, we investigated the expression level of apoptosis and autophagy-related proteins in different passages of PD-MSCs using Western blot analysis. The expression of apoptotic molecule Bax was markedly increased $(P$ value $=$ 0.008), whereas antiapoptotic $\mathrm{Bcl}-2$ expression was similar in both late and early passage PD-MSCs. Overexpression

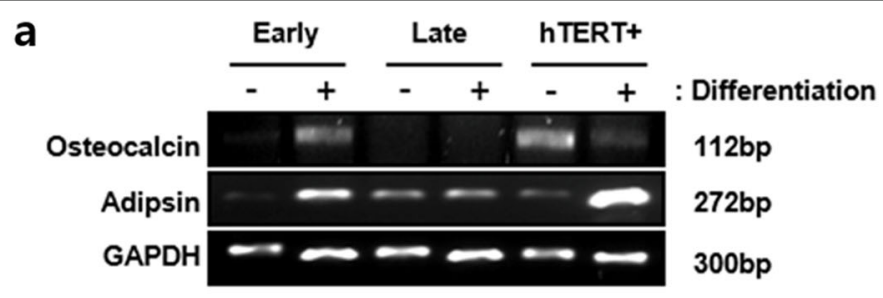

b

Osteogenesis
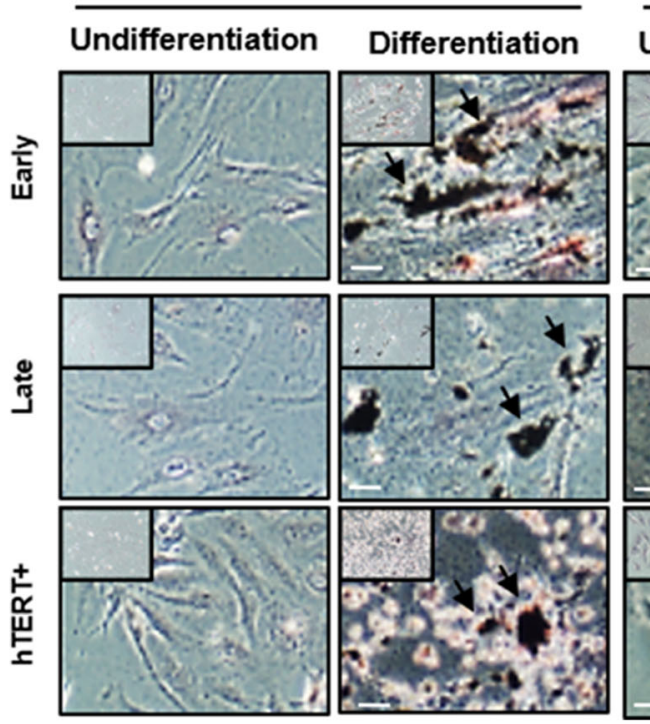

Differentiation
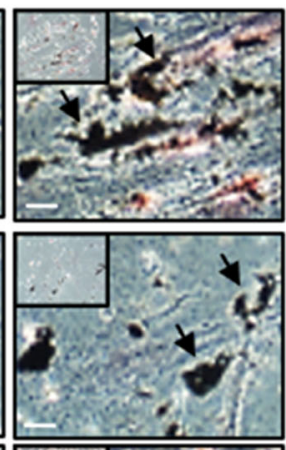

Adipogenesis
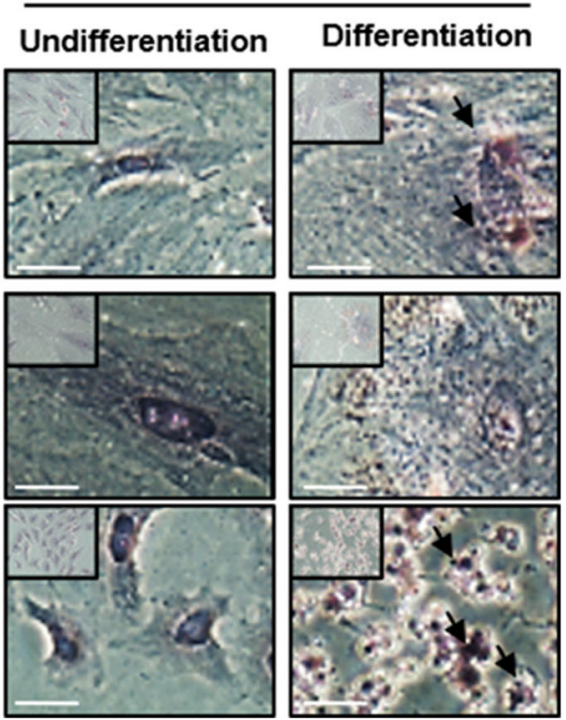

Fig. 2 Differentiation potential of senescent PD-MSCs. a After early and late passage PD-MSCs and hTERT+ were differentiated for 2 weeks, the expression of genes related to osteogenesis and adipogenesis was measured by RT-PCR. $\mathbf{b}$ Osteogenic and adipogenic lineages were determined by von Kossa (magnification, $\times 100$ ) and Oil-Red O staining (magnification, $\times 200$ ), respectively. The data were representative of three independent experiments 


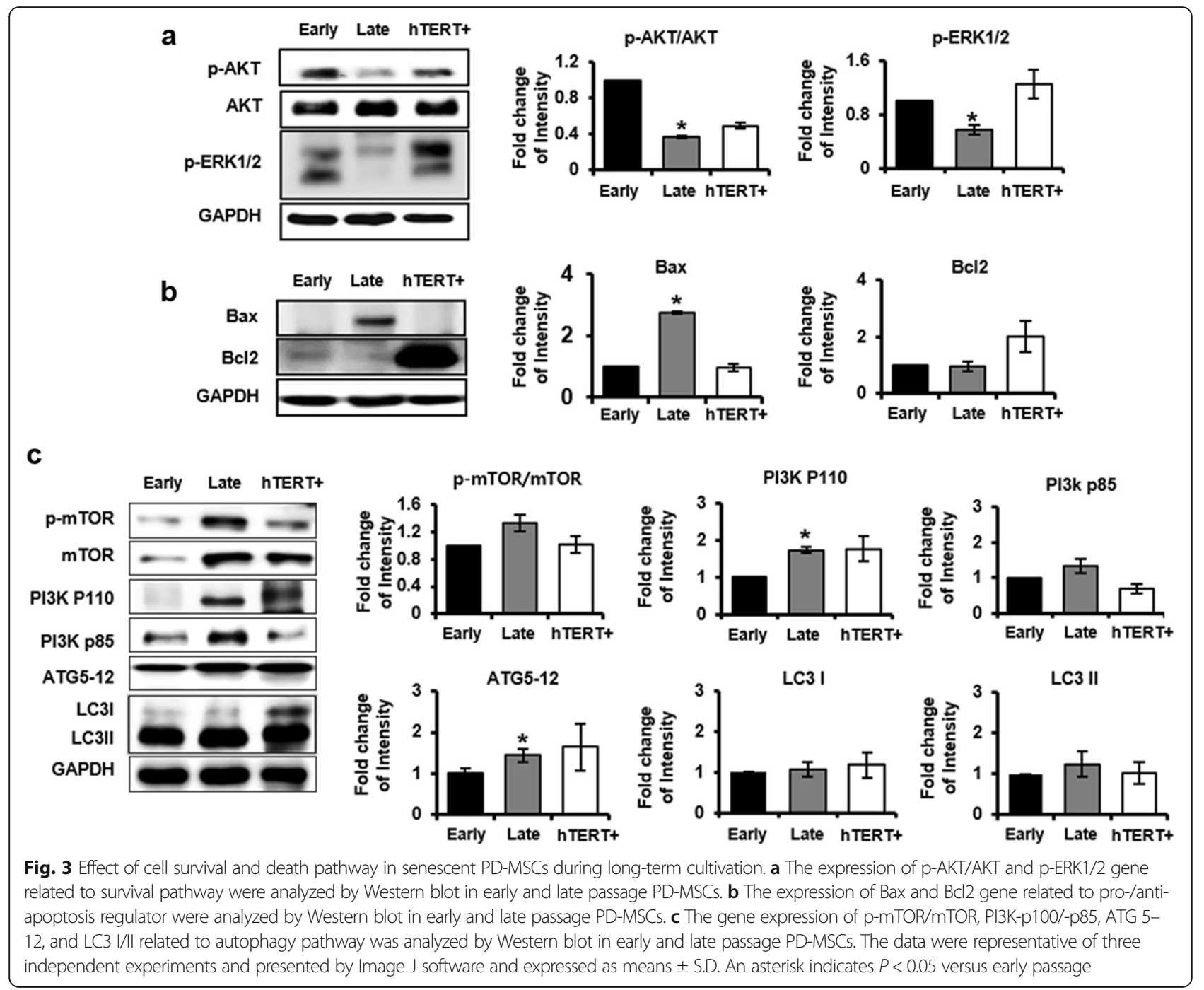

of Bcl-2 in hTERT+ PD-MSCs was observed to prevent cell death (Fig. 3b). Several autophagy-related proteins act in cells during aging. Mammalian target of rapamycin (mTOR) is a negative regulator of autophagy. As shown in Fig. 3c, the p-mTOR/mTOR level was slightly increased in late passage, and also downstream factors, PI3K and ATG5-12 $(P$ value $=0.02)$, were increased, but slight increasing trend of LC3I/II level showed no significant statistical differences between early and late passages. These results suggest that senescent PD-MSCs according to long-term culture represent the possibility of affecting the initial process of autophagy. Therefore, further studies are required to clarify the mechanism of autophagy regulation in senescent PD-MSCs.

Effect of mitochondrial dysfunction in senescent PD-MSCs during long-term cultivation

Since it is well known that cellular senescence has been associated with alteration of mitochondrial function by oxidative stress such as reactive oxygen species (ROS) [10], we observed whether long-term cultivation would promote ROS production in PD-MSCs. The level and localization of ROS production in the mitochondria was determined by double staining with MitoTracker Green, which labels the mitochondria in a manner that is independent of the membrane potential, and MitoSox Red, which specifically stains mitochondrial superoxide $\left(\mathrm{O}_{2}^{-}\right)$among ROS. As shown in Fig. 4a, it was confirmed that ROS was generated in the mitochondrial region by fluorescent overlap in these PDMSCs. In late passage PD-MSCs, the ROS production as well as mitochondrial mass increased. To further clarify the ROS level, $\mathrm{H}_{2} \mathrm{O}_{2}$ accumulation was quantitatively examined by fluorescence microplate reader using DCFDA, which is a non-fluorescent DCFDA form a fluorescent DCF in the presence of ROS, especially $\mathrm{H}_{2} \mathrm{O}_{2}$. Consistently, late passage PD-MSCs showed about a 1.5-fold increase, whereas hTERT+ PD-MSCs showed a significant decrease in compared to early PD-MSCs (Fig. 4b). Next, we measured 


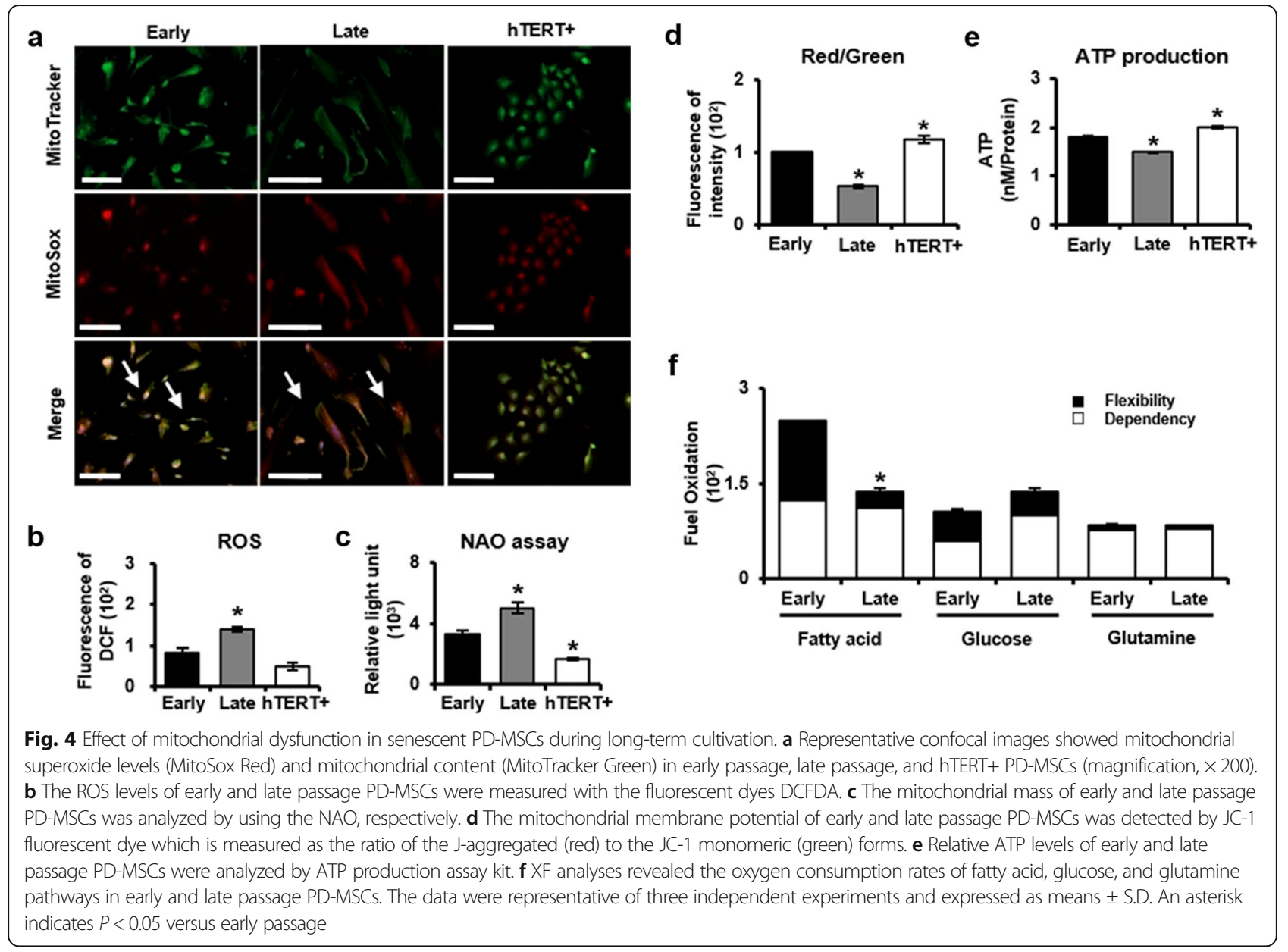

mitochondrial biogenesis in early and late passage PDMSCs. To quantitatively clarify mitochondrial mass, we used NAO, which measures mitochondrial mass by binding to cardiolipin in all mitochondria. Similar to the morphology identified by MitoTracker, mitochondrial mass increased in late passage PD-MSCs compared with early cells (Fig. 4c). We then evaluated mitochondria metabolic functions by mitochondrial membrane potential $(\Delta \psi \mathrm{m})$ and ATP production assays (Fig. 4d, e). Mitochondrial membrane potential was quantified by the JC-1 fluorescence dye. At low membrane potential, a cationic carbocyanine dye accumulates as a monomer in the mitochondria, which yield a green fluorescence (depolarization), while it aggregates at high membrane potential with a red fluorescence (hyperpolarization). Our results showed about 50\% reduction (i.e., depolarization) in $\Delta \psi \mathrm{m}$ as a ratio of red/ green fluorescence intensity in late passage PD-MSCs in comparison to early cells. However, hTERT overexpressed PD-MSCs showed higher hyperpolarization than early cells. Cellular ATP content was also decreased in late PD-MSCs similar to $\Delta \psi \mathrm{m}$. Mitochondria produce ATP through electron transport chain (ETC) and oxidative phosphorylation (OXPHOS) in three major nutrients such as fatty acids, glucose, and amino acids. A "metabolic flexible" means free switch between major nutrients depending on nutritional and physiological cues [19]. A previous study demonstrated that senescent bone marrow MSCs evidenced metabolic inflexibility [20]. Accordingly, we investigated the changes in energy supply and metabolic flexibility during long-term cultivation in PD-MSCs by the XF Mito Fuel Flex system. The metabolic flexibility in media containing each major nutrient is low in replicative senescent PD-MSCs compared to early cells. Changes in the flexibility of fatty acids and glucose media in early PD-MSCs were more dynamic than those of glutamine, and dependency in these media was also consistent in late PD-MSCs, suggesting that periodic shifts in fatty acids and glucose are responsible for energy metabolism of PD-MSCs (Fig. 4f). Taken together, these results suggest that long-term cultivation causes mitochondrial dysfunction in mitochondrial membrane potential and metabolic flexibility following the morphological changes of mitochondria in PD-MSCs. 
Effect of increased CPT1A in senescent PD-MSCs during long-term cultivation

We investigated fatty acid oxidation (FAO)-related factors to confirm FAO pathway in PD-MSCs during long-term cultivation. In skeletal muscle cells, it is well established that AMP-activated protein kinase (AMPK) inhibits acetyl-CoA carboxylase (ACC) through phosphorylation, which reduces intracellular malonyl-CoA levels and stimulates carnitine palmitoyl transferase 1 (CPT1) and then increases the influx of long-chain fatty acids into the mitochondria where they are oxidized [21]. Quantitative RTPCR revealed that late passage PD-MSCs increased dramatically the mRNA levels of ACC and CPT1A compared to early cells (Fig. 5a). In addition, Western blot analysis showed that the increase of CPT1A $(P$ value $=0.06)$ was followed by the increase of expression of $\mathrm{p}$-ACC versus total-ACC by long-term cultivation in PD-MSCs ( $P$ value $=0.053$ ), while the phosphorylation level of AMPK, an upstream of ACC, was significantly decreased suggesting activation of AMPK is cell type-specific. Peroxisome proliferator-activated receptor (PPAR $\alpha)$ is another major regulator of FAO as a transcription factor, which is predominantly expressed in tissues that oxidize fatty acids at a rapid rate, such as the liver, brown adipose tissue, heart, and kidney [22]. As expected, increased passages of PDMSCs induced a significant increase of PPAR $\alpha(P$ value $=$ 0.02) (Fig. 5b). To further clarify the role of CPT1A increased by replicative senescent in PD-MSCs, we blocked CPT1A with the pharmacologic CPT1 inhibitor etomoxir or siRNA targeting CPT1A in late passage PDMSCs and evaluated its effects on FAO signaling and senescence biomarker expression. We confirmed that the mRNA expressions of CPT1A and ACC were markedly inhibited, and the protein expression level of p-ACC as well as CPT1A was also reduced by treatment with etomoxir or siRNA in late passage PD-MSCs (Additional file 1: Figures S2a and S2b). Interestingly, inhibition of CPT1A reduced significantly SA- $\beta$-gal positive cells, indicating that fatty acid metabolism plays an important role in replicative senescence of PD-MSCs (Fig. 5c).

\section{Effect of downregulated CPT1A on mitochondrial activities in senescent PD-MSCs}

To further specifically look at the importance of FAO metabolism in PD-MSCs, we subsequently investigated how the mitochondrial metabolic function changes by CPT1 inhibitor treatment in late passage PD-MSCs. Interestingly, treatment of CPT1A siRNA decreased ATP production despite the reduction of mitochondrial mass and ROS, including an increase of mitochondrial membrane potential, suggesting that fatty acid metabolism of PDMSCs during long-term cultivation contributes more to ATP production than glycolysis (Fig. 6a-d). The glycolytic ability of senescent PD-MSCs was performed using XF24

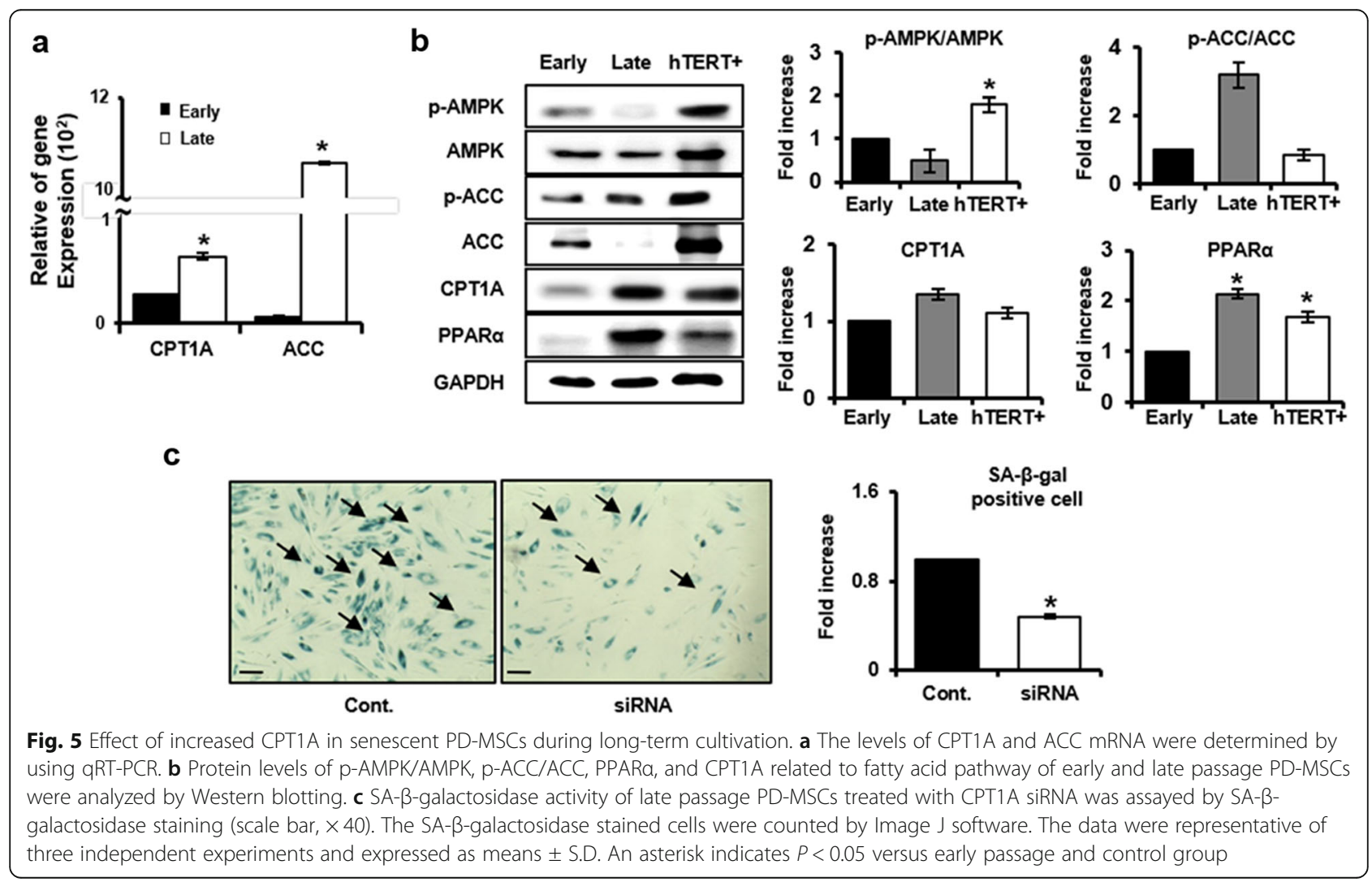




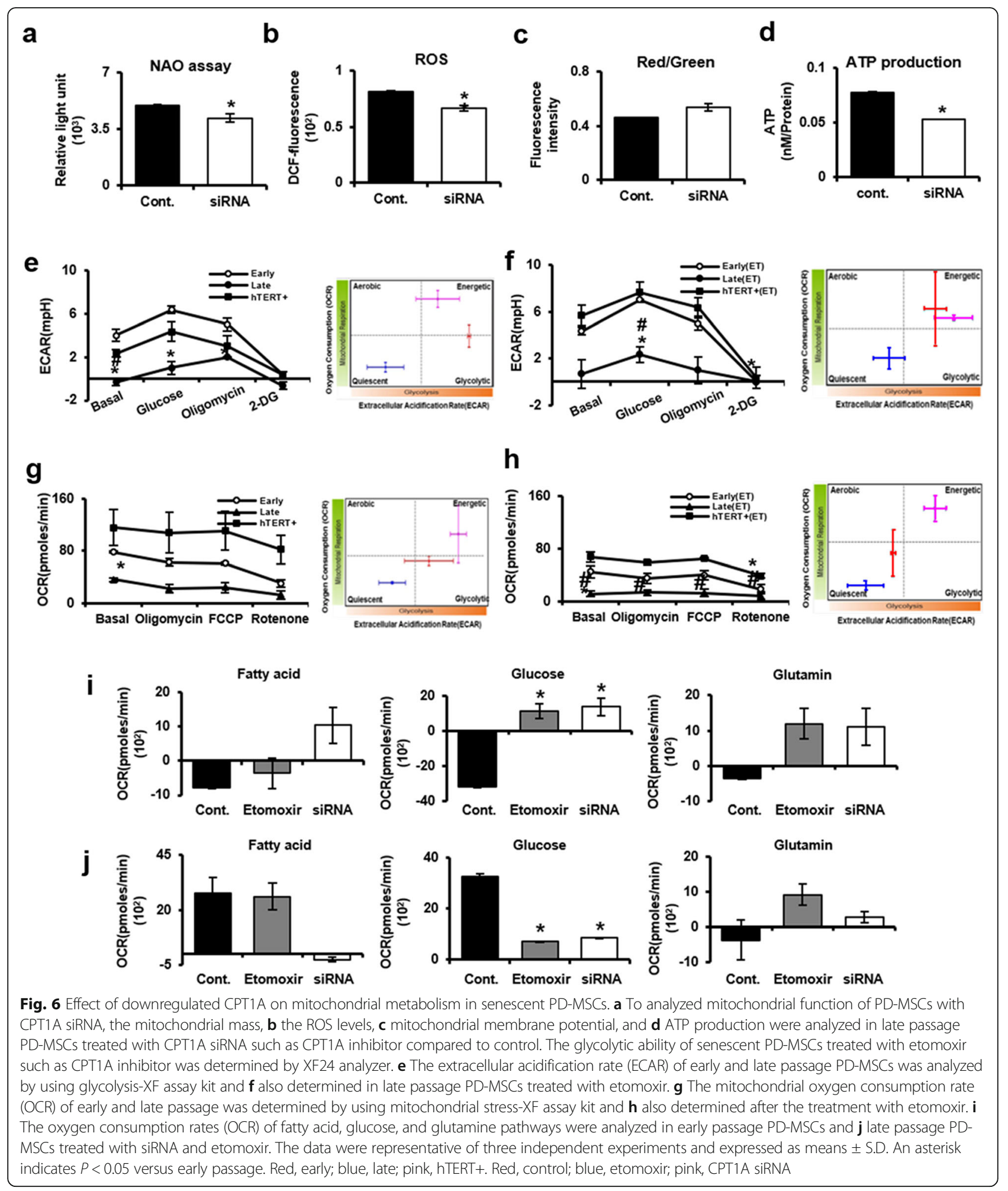

Extracellular Flux analyzer. Oligomycin inhibits mitochondrial ATP and 2-DG inhibits glycolysis. So, XF-assay analyzed cells' dependence on mitochondrial energy metabolism including glycolysis pathway. The glycolytic rate in late passage was significantly lower than those in early passage and hTERT+ PD-MSCs (Fig. 6e). We also confirmed decreased glycolytic activity in late passage after the treatment with etomoxir compared to early passage 
(Fig. 6f). Mitochondrial stress test was also performed to measure oxygen consumption rate (OCR) of senescent PD-MSCs. The results showed that late passage PD-MSCs have statistically significant lower OCR than early passage regardless of the treatment with etomoxir (Fig. 6g, h). These findings suggest that senescent PD-MSCs are associated with altered mitochondrial metabolism including fatty acid oxidation and glycolysis. Furthermore, the alterations in mitochondrial fuel usage, such as fatty acids, glucose, and glutamine, were determined by using XF Mito Fuel Flex system. Otherwise, in late passage PD-MSCs (Fig. 6j), the OCRs in fatty acid and glucose pathways except glutamine pathway were higher than those of early passage (Fig. 6i). Interestingly, etomoxir treatment on fatty acid pathway in late passage markedly increased the OCR level, whereas the knockdown of CPT1A with siRNA on fatty acid pathway in late passage significantly decreased the OCR level compared to early passage. These results suggest that fatty acid is the most influential factor among alterations in mitochondrial fuel usage, and the expression of CPT1A plays an important role in mitochondrial function. In addition, CPT1A can lower the OCR which is the mitochondrial stress level in senescent PD-MSCs.

\section{Discussion}

The limited self-renewal of MSCs is one of obstacles to overcome in the development of stem cell-based therapy in degenerative medicine, although they have multi-lineage differentiation potential and immunomodulatory effect [23]. Cellular stresses can trigger shortness of hTERT in cells and induce replicative senescence. Accordingly, to investigate cellular senescence and related mechanism during long-term cultivation in PD-MSCs, we used PD-MSCs with different passages and hTERT+ PD-MSCs established in our previous study [24] as a positive control. In our present study, we demonstrated that PD-MSCs during long-term cultivation undergo senescence, which is characterized by a lowered differentiation potential and a reduced proliferation rate by $\mathrm{G} 1$ cell cycle arrest via p21 in a p53 independent pathway with the same result as previous research [25], although it differs from some studies that the $\mathrm{p} 53 / \mathrm{p} 21$ pathway plays a role in cell growth arrest, cellular senescence, and apoptosis [26, 27]. Several studies reported that overexpression of hTERT helps PD-MSCs escape from replicative senescence suggesting that hTERT plays an important role in replicative senescence. Interestingly, the differentiation potential of hTERT+ has a difference for adipogenic and osteogenic compared to other cells. In previous reports, Ikebale et al. reported that immortalized stem cells by TERT gene transfection shows lower potential for adipogenic differentiation [28, 29]. These data we matched with our results as Fig. $2 \mathrm{~b}$ in the study.

Mitochondria are highly dynamic organelles, which can regulate through the process of fission and fusion, allowing them to adjust their size and shape during apoptosis, autophagy, mitochondrial biogenesis, adipogenic differentiation of human mesenchymal stem cells (hMSCs), and cellular senescence $[10,30]$. Senescence can be caused by cellular stresses, especially, ROS-mediated oxidative stress induces replicative senescence leading to lipid peroxidation, mitochondrial dysfunction, energy failure, and metabolic disturbance in the cell membrane [31, 32]. Mitochondria are also energy factories to maintain and regulate various cellular processes and their functions are critical to cell survival [33]. In the mitochondrial fuel selection, glucose has been generally regarded as the primary energy source rather than lipids and amino acids, as glucose supply is constant, consistent, and reliable. However, fatty acid metabolism has been found to play an important role during human pregnancy and in placental growth by the activity and expression confirmation of FAO enzymes in the human placenta [[17]. Recently, it has been also reported that fatty acid metabolism is associated with the aging of organelles with aging and the senescence of bone marrow MSCs [20, 34]. Similarly, our study demonstrated that metabolic activity of fatty acids was higher than that of glucose or glutamine in both early and late passages, but late passage PD-MSCs exhibited inflexible compared with early passage cells. To specify more details, we investigated signaling pathway underlying FAO. As expected, the PPAR $\alpha$, p-ACC, and CPT1A associated with fatty acid metabolism increased in late passage. Interestingly, the CPT1A knockdown reversed mitochondrial dysfunction (decreased ROS, NAO, and improved mitochondrial membrane potential) and inhibited senescence induced by longterm cultivation in PD-MSCs, indicating that increased CPT1A in senescent PD-MSCs triggers mitochondrial dysfunction by unbalanced energy metabolism as well as antiaging effect by downregulated CPT1A gene and that FAO has a significant impact on the senescence of PD-MSCs. This is similar to the previous reports that the specific increase in saturated fatty acids is a characteristic of many agerelated human diseases including cardiovascular disease and cancer, and that saturated fatty acid metabolism is key link between cell division, cancer, and senescence in cellular and whole organism aging [34, 35]. However, ATP production did not restore as much as early PD-MSCs despite the increased mitochondrial membrane potential. It is considered that the replicative senescent PD-MSCs have relatively metabolic inflexibility compared to early passage cells. In addition, the inhibition of FAO with etomoxir or CPT1A siRNA in replicative senescent PD-MSCs showed no change in glutamine metabolism and glycolysis shifting for energy production, which means that they enable exquisite crosstalk and cooperation between fatty acid and glucose to maintain 
energy as in Randle's glucose-fatty acid cycle hypothesis (Additional file 1: Figure S3a, b, c, d and e) [36]. Some studies have indicated an increase in glucose uptake during oncogene-induced senescence (OIS), and cancer cells preferentially use glycolysis under aerobic conditions [37, 38]. Accordingly, it may also be thought that higher aerobic glycolysis than anaerobic during long-term cultivation promotes senescence of PD-MSCs, although it is cell typespecific. Further studies on metabolic alterations are needed to clearly understand the senescent process of PD-MSCs. Taken together, our findings first showed that CPT1A plays an important factor in mitochondria function via regulation of energy metabolism, and ROS level in replicative senescence of PD-MSCs according to long-term cultivation. These data help us understand the fundamental mechanism of self-renewal of PD-MSCs and support to overcome the replicative senescence of PD-MSCs.

\section{Conclusions}

In this study, PD-MSCs during prolonged in vitro culture undergo senescence exhibiting reduced proliferation rate by G1 arrest and lowered differentiation potential, and overexpression of hTERT helps PD-MSCs escape from replicative senescence. Also, senescent PD-MSCs according to long-term culture represent the possibility of affecting the initial process of autophagy. Senescent PD-MSCs appeared mitochondrial dysfunction in mitochondrial membrane potential and metabolic flexibility following the morphological changes of mitochondria. In addition, CPT1A decreased the OCR which is the mitochondrial stress level in senescent PD-MSCs. Therefore, our findings showed that CPT1A plays a role as an essential factor in mitochondria function via control of energy metabolism, and ROS level in replicative senescence of PD-MSCs through long-term cultivation.

\section{Supplementary information}

Supplementary information accompanies this paper at https://doi.org/10. 1186/s13287-019-1471-y.

Additional file 1: Figure S1. S1Characterization related to tumor suppressor gene expression in PD-MSCs during long-term cultivation. The p53 gene expression related to tumor suppressor in Early and Late passage PD-MSCs was assayed by western blotting. The data were representative of three independent experiments and expressed as means \pm S.D. ${ }^{*}$ indicates $P<0.05$ versus Early passage. Figure S2. Effect of fatty acids in senescent PD-MSCS according to CPT1A inhibition. a The levels of CPT1A and ACC mRNA were analyzed in Late passage PD-MSCs with Etomoxir and siRNA-CPT1A treated group by using qRT-PCR. b The protein levels of p-ACC/ACC ratio and CPT1A were assayed in Late passage PD-MSCs treated with Etomoxir by using western blotting. The data were representative of three independent experiments and expressed as means \pm S.D. ${ }^{*}$ indicates $P<0.05$ versus Non-treated Late passage PD-MSCs. Figure S3. Effect of mitochondrial metabolism in PDMSCs according to CPT1A inhibition through Etomoxir treatment. a The Extracellular acidification rate (ECAR) of Early and Late passage PD-MSCS were analyzed by using glycolysis-XF assay. b Mitochondrial oxygen consumption (OCR) of Early and Late passage PD-MSCs were analyzed by using mitochondrial stress-XF assay. c The mitochondrial fuel levels of senescent PD-MSCs with siRNA CPT1A were analyzed according to inhibition of fatty acid, $\mathbf{d}$ glucose and $\mathbf{e}$ glutamic pathway by using XF24 analyzer. The data were representative of three independent experiments and expressed as means \pm S.D. ${ }^{*}$ indicates $P<0.05$ versus Non-treated group. ${ }^{* *}$ indicates $p>0.05$ versus in group (e.g., control and siRNA).

\section{Abbreviations}

ACC: Acetyl-CoA carboxylase; CPT1: Carnitine palmitoyl transferase 1; ETC: Electron transport chain; FAO: Fatty acid oxidation; hMSCs: Human mesenchymal stem cells; OCR: Oxygen consumption rate; OIS: Oncogeneinduced senescence; OXPHOS: Oxidative phosphorylation; PD-MSCs: Human placenta-derived mesenchymal stem cells; PPARa: Peroxisome proliferatoractivated receptor; ROS: Reactive oxygen species; SA- $\beta$-Gal: Senescenceassociated $\beta$-galactosidase

\section{Acknowledgements}

The authors would like to thank Dr. Jong Ho Choi (University of Northwestern) for his assistance with flow cytometry and Hyun Jung Lee (CHA medical) for her support with adipoblast/osteoblast differentiation.

\section{Authors' contributions}

JS and HSJ did the analysis and interpretation of data and manuscript drafting. SP did the interpretation of data and manuscript drafting. JYK and JOL did the data analysis. CJK helped in the critical discussion. GJK conceived and designed the experiments and directed the manuscript drafting, financial support, and final approval of the manuscript. All authors read and approved the final manuscript.

\section{Funding}

This research was supported by a grant of the Korea Health Technology R\&D Project through the Korea Health Industry Development Institute (KHIDI), funded by the Ministry of Health \& Welfare, Republic of Korea (grant number: HI17C1050) and a grant from the National Research Foundation of Korea (NRF) funded by the Ministry of Science, ICT \& Future Planning, Republic of Korea (grant number: NRF-2017M3A9B406).

\section{Availability of data and materials}

The data that support the findings of this study are available from the corresponding author upon reasonable request.

\section{Ethics approval and consent to participate}

This study was conducted under the guidelines and with the approval of the affiliated Institutional Review Board of the Gangnam CHA General Hospital, Seoul, Korea. All patients consented to the respective use of their tissues.

\section{Consent for publication}

Not applicable

\section{Competing interests}

The authors declare that they have no competing interests.

\section{Author details}

'Department of Biomedical Science, CHA University, 689, Sampyeong-dong, Bundang-gu, Seongnam-si, Gyeonggi-do, Republic of Korea. ${ }^{2}$ Department of Anatomy, Korea University College of Medicine, Seoul, Republic of Korea. ${ }^{3}$ Department of Pathology, University of Ulsan College of Medicine, Asan Medical Center, Seoul, Republic of Korea.

Received: 30 April 2019 Revised: 10 October 2019 Accepted: 25 October 2019 Published online: 03 January 2020

\section{References}

1. Hass R, Kasper C, Bohm S, Jacobs R. Different populations and sources of human mesenchymal stem cells (MSC): a comparison of adult and neonatal tissue-derived MSC. Cell Commun Signal. 2011;9:12.

2. Kim GJ. Advanced research on stem cell therapy for hepatic diseases: potential implications of a placenta-derived mesenchymal stem cell-based strategy. Hanyang Med Rev. 2015;35(4):207-14. 
3. Hayflick L, Moorhead PS. The serial cultivation of human diploid cell strains Exp Cell Res. 1961;25:585-621.

4. Sethe S, Scutt A, Stolzing A. Aging of mesenchymal stem cells. Ageing Res Rev. 2006;5(1):91-116.

5. Boyette $L B$, Tuan RS. Adult stem cells and diseases of aging. J Clin Med. 2014;3(1):88-134.

6. Gerland LM, Peyrol S, Lallemand C, Branche R, Magaud JP, Ffrench M. Association of increased autophagic inclusions labeled for betagalactosidase with fibroblastic aging. Exp Gerontol. 2003;38(8):887-95.

7. Zheng Y, Hu CJ, Zhuo RH, Lei YS, Han NN, He L. Inhibition of autophagy alleviates the senescent state of rat mesenchymal stem cells during longterm culture. Mol Med Rep. 2014;10(6):3003-8.

8. Gewirtz DA. Autophagy and senescence: a partnership in search of definition. Autophagy. 2013;9(5):808-12.

9. Goehe RW, Di X, Sharma K, Bristol ML, Henderson SC, Valerie K, Rodier F, Davalos AR, Gewirtz DA. The autophagy-senescence connection in chemotherapy: must tumor cells (self) eat before they sleep? J Pharmacol Exp Ther. 2012;343(3):763-78.

10. Ziegler DV, Wiley CD, Velarde MC. Mitochondrial effectors of cellular senescence: beyond the free radical theory of aging. Aging Cell. 2015;14(1):1-7.

11. Hsu YC, Wu YT, Yu TH, Wei YH. Mitochondria in mesenchymal stem cell biology and cell therapy: From cellular differentiation to mitochondrial transfer. Semin Cell Dev Biol. 2016;52:119-31.

12. Finley LW, Haigis MC. The coordination of nuclear and mitochondrial communication during aging and calorie restriction. Ageing Res Rev. 2009; 8(3):173-88

13. Bratic I, Trifunovic A. Mitochondrial energy metabolism and ageing. Biochim Biophys Acta. 2010;1797(6-7):961-7.

14. Kim YM, Shin HT, Seo YH, Byun HO, Yoon SH, Lee IK, Hyun DH, Chung HY, Yoon G. Sterol regulatory element-binding protein (SREBP)-1-mediated lipogenesis is involved in cell senescence. J Biol Chem. 2010;285(38):29069-77.

15. Henique C, Mansouri A, Fumey G, Lenoir V, Girard J, Bouillaud F, Prip-Buus C, Cohen I. Increased mitochondrial fatty acid oxidation is sufficient to protect skeletal muscle cells from palmitate-induced apoptosis. J Biol Chem. 2010;285(47):36818-27.

16. Zammit VA. Carnitine palmitoyltransferase 1: central to cell function. IUBMB Life. 2008;60(5):347-54.

17. Shekhawat P, Bennett MJ, Sadovsky Y, Nelson DM, Rakheja D, Strauss AW. Human placenta metabolizes fatty acids: implications for fetal fatty acid oxidation disorders and maternal liver diseases. Am J Physiol Endocrinol Metab. 2003;284(6):E1098-105.

18. Lee MJ, Jung J, Na KH, Moon JS, Lee HJ, Kim JH, Kim Gl, Kwon SW, Hwang SG, Kim GJ. Anti-fibrotic effect of chorionic plate-derived mesenchymal stem cells isolated from human placenta in a rat model of CCl (4)-injured liver: potential application to the treatment of hepatic diseases. J Cell Biochem. 2010;111(6):1453-63.

19. Muoio DM. Metabolic inflexibility: when mitochondrial indecision leads to metabolic gridlock. Cell. 2014;159(6):1253-62.

20. Capasso S, Alessio N, Squillaro T, Di Bernardo G, Melone MA, Cipollaro M, Peluso G, Galderisi U. Changes in autophagy, proteasome activity and metabolism to determine a specific signature for acute and chronic senescent mesenchymal stromal cells. Oncotarget. 2015;6(37):39457-68.

21. Yoon MJ, Lee GY, Chung JJ, Ahn YH, Hong SH, Kim JB. Adiponectin increases fatty acid oxidation in skeletal muscle cells by sequential activation of AMP-activated protein kinase, p38 mitogen-activated protein kinase, and peroxisome proliferator-activated receptor alpha. Diabetes. 2006; 55(9):2562-70.

22. Monsalve FA, Pyarasani RD, Delgado-Lopez F, Moore-Carrasco R. Peroxisome proliferator-activated receptor targets for the treatment of metabolic diseases. Mediators Inflamm. 2013;2013:549627.

23. Kim MJ, Shin KS, Jeon JH, Lee DR, Shim SH, Kim JK, Cha DH, Yoon TK, Kim GJ. Human chorionic-plate-derived mesenchymal stem cells and Wharton's jelly-derived mesenchymal stem cells: a comparative analysis of their potential as placenta-derived stem cells. Cell Tissue Res. 2011;346(1):53-64.

24. Lee HJ, Choi JH, Jung J, Kim JK, Lee SS, Kim GJ. Changes in PTTG1 by human TERT gene expression modulate the self-renewal of placentaderived mesenchymal stem cells. Cell Tissue Res. 2014;357(1):145-57.

25. Ho PJ, Yen ML, Tang BC, Chen CT, Yen BL. H2O2 accumulation mediates differentiation capacity alteration, but not proliferative decline, in senescent human fetal mesenchymal stem cells. Antioxid Redox Signal. 2013;18(15): 1895-905.
26. Bieging KT, Mello SS, Attardi LD. Unravelling mechanisms of p53-mediated tumour suppression. Nat Rev Cancer. 2014;14(5):359-70.

27. Gu Z, Jiang J, Tan W, Xia Y, Cao H, Meng Y, Da Z, Liu H, Cheng C. p53/p21 Pathway involved in mediating cellular senescence of bone marrow-derived mesenchymal stem cells from systemic lupus erythematosus patients. Clin Dev Immunol. 2013:2013:134243.

28. Ikbale el A, Goorha S, Reiter LT, Miranda-Carboni GA. Effects of hTERT immortalization on osteogenic and adipogenic differentiation of dental pulp stem cells. Data Brief. 2016;6:696-9.

29. Liu TM, Ng WM, Tan HS, Vinitha D, Yang Z, Fan JB, Zou Y, Hui JH, Lee EH, Lim B. Molecular basis of immortalization of human mesenchymal stem cells by combination of p53 knockdown and human telomerase reverse transcriptase overexpression. Stem Cells Dev. 2013;22(2):268-78.

30. Zhang Y, Marsboom G, Toth PT, Rehman J. Mitochondrial respiration regulates adipogenic differentiation of human mesenchymal stem cells. PLoS One. 2013;8(10):e77077.

31. Ortuno-Sahagun D, Pallas M, Rojas-Mayorquin AE. Oxidative stress in aging: advances in proteomic approaches. Oxid Med Cell Longev. 2014;2014: 573208.

32. Babizhayev MA, Yegorov YE. Tissue formation and tissue engineering through host cell recruitment or a potential injectable cell-based biocomposite with replicative potential: molecular mechanisms controlling cellular senescence and the involvement of controlled transient telomerase activation therapies. J Biomed Mater Res A. 2015;103(12):3993-4023.

33. Amigo I, da Cunha FM, Forni MF, Garcia-Neto W, Kakimoto PA, LuevanoMartinez LA, Macedo F, Menezes-Filho SL, Peloggia J, Kowaltowski AJ. Mitochondrial form, function and signalling in aging. Biochem J. 2016; 473(20):3421-49.

34. Ford JH. Saturated fatty acid metabolism is key link between cell division, cancer, and senescence in cellular and whole organism aging. Age (Dordr). 2010;32(2):231-7.

35. Volpe JJ, Vagelos PR. Mechanisms and regulation of biosynthesis of saturated fatty acids. Physiol Rev. 1976;56(2):339-417.

36. Randle PJ. Regulatory interactions between lipids and carbohydrates: the glucose fatty acid cycle after 35 years. Diabetes Metab Rev. 1998; 14(4):263-83.

37. Moiseeva O, Bourdeau V, Roux A, Deschenes-Simard X, Ferbeyre G. Mitochondrial dysfunction contributes to oncogene-induced senescence. Mol Cell Biol. 2009;29(16):4495-507.

38. Aird KM, Zhang R. Metabolic alterations accompanying oncogene-induced senescence. Mol Cell Oncol. 2014;1(3):e963481.

\section{Publisher's Note}

Springer Nature remains neutral with regard to jurisdictional claims in published maps and institutional affiliations.

Ready to submit your research? Choose BMC and benefit from:

- fast, convenient online submission

- thorough peer review by experienced researchers in your field

- rapid publication on acceptance

- support for research data, including large and complex data types

- gold Open Access which fosters wider collaboration and increased citations

- maximum visibility for your research: over $100 \mathrm{M}$ website views per year

At BMC, research is always in progress.

Learn more biomedcentral.com/submissions 